\title{
T regulatory lymphocytes in patients with endometriosis
}

\author{
MAREK GOGACZ ${ }^{1}$, IZABELA WINKLER ${ }^{1}$, AGNIESZKA BOJARSKA-JUNAK ${ }^{2}$, \\ JACEK TABARKIEWICZ ${ }^{2}$, ANDRZEJ SEMCZUK ${ }^{1}$, TOMASZ RECHBERGER ${ }^{1}$ and ANETA ADAMIAK ${ }^{1}$ \\ ${ }^{1}$ Second Department of Gynaecology, Medical University of Lublin, Lublin 20-954; \\ ${ }^{2}$ Department of Clinical Immunology, Medical University, Lublin 20-093, Poland
}

Received October 14,2013; Accepted March 25, 2014

DOI: $10.3892 / \mathrm{mmr} .2014 .2294$

\begin{abstract}
The present study aimed to investigate the presence of $\mathrm{T}$ regulatory cells (Tregs) in the peripheral blood (PB) and peritoneal fluid $(\mathrm{PF})$ in females with endometriosis. The present study included 42 patients who underwent laparoscopy between 2010 and 2011. Flow cytometry was used to determine the percentage of Tregs in the PF and PB of the patients. No significant difference was observed in the percentage of Tregs in the patients in the endometriosis group compared with those in the control group in the $\mathrm{PF}(9.1 \pm 5.4$ vs. $9.1 \pm 3.8 \%)$ or the $\mathrm{PB}$ (6.5 \pm 3.2 vs. $6.5 \pm 3.7 \%)$. However, the percentage of Tregs was found to be higher in the PF compared with the PB in the patients in the endometriosis and control groups, but significance was found only in those in the control group. Furthermore, no significant difference was observed in the Treg concentration in the patients with early stage (I/II) endometriosis compared with those with late stage (III/IV) endometriosis. Moreover, no significant correlation was found between the percentage of Tregs and the white blood cell count, lymphocyte count or CA125 concentration in the patients. These findings suggest that the local host-defense mechanism is deficient in patients with endometriosis, thus endometriosis should not be treated as an autoimmune condition.
\end{abstract}

\section{Introduction}

Endometriosis is an estrogen-dependent disorder characterized by the growth of endometrial tissue outside of the uterine cavity. This is a common gynecological disorder, which affects

Correspondence to: Izabela Winkler, Second Department of Gynaecology, Medical University of Lublin, 8 Jaczewski Street, Lublin 20-954, Poland

E-mail: ikochans@interia.pl

Abbreviations: DC, dendritic cells; E, endometriosis group; FOXP3, forkhead box P3; NK, natural killer; PF, peritoneal fluid; $\mathrm{PB}$, peripheral blood; rASRM, revised American Society for Reproductive Medicine; Treg, T regulatory; TGF- $\beta$, transforming growth factor $\beta$

Key words: Treg cells, endometriosis, peritoneal fluid, peripheral blood up to $10 \%$ of females at reproductive age (1). Endometriosis has been proposed to be caused by immune system impairments (2). Endometriosis is associated with local and systemic immune changes. During endometriosis, levels of peritoneal macrophages and the secretion of proinflammatory cytokines and growth factors are increased. Moreover, the activation of natural killer (NK) cells is reduced. The pathogenesis of endometriosis may involve various autoantibodies, including anti-nuclear and -phospholipid antibodies $(1,3,4,5)$. Thus, it has been suggested that endometriosis is an autoimmune disease $(5,6)$.

Regulatory T cells (Tregs) were first identified in 1995 by Sakaguchi et al (7) and have been reported to represent $~ 10 \%$ of peripheral cluster of differentiation (CD) $4^{+} \mathrm{T}$ helper cells $(7,8)$. Tregs are characterized by the presence of surface markers, including CD4, CD25 and forkhead box P3 (FOXP3). Tregs are responsible for the regulation of immune processes and the development and maintenance of immunological tolerance through active suppression. Tregs have been reported to release suppressor cytokines, including interleukin (IL)-10 and transforming growth factor (TGF)- $\beta$. These cytokines reduce the activity of effector $\mathrm{T}$ cells. Tregs have been found to suppress various immune activities, including macrophage activation, T- and B-cell proliferation, dendritic cell (DC) and NK cell functions, mast cell degranulation and cytokine release $(9,10)$.

Tregs are involved in the pathogenesis of autoimmune disorders, including thyroiditis, inflammatory bowel disease, multiple sclerosis, myasthenia gravis, systemic lupus erythematosus, type I diabetes mellitus, rheumatoid arthritis, psoriasis, autoimmune gastritis, polyendocrinopathy and enteropathy. However, the role of Tregs in endometriosis has yet to be elucidated $(10,11,12)$.

In this study, we examined percentage of lymphocyte Tregs in peripheral blood (PB) and peritoneal fluid (PF) in patients with endometriosis. Moreover, we observed the correlation between the percentage of Tregs with varying severity of endometriosis and white blood cell counts.

\section{Materials and methods}

Materials. PB was obtained prior to general anesthesia from the antecubital vein and PF was obtained during laparoscopy with anesthetic (Desfluran, Baxter, Deerfield, IL, USA; Diprivan, AstraZeneca, London, UK; Thiopental, Sandoz, Warsaw, Poland; Fentanyl, Polfa, Warsaw, Poland; Scoline, 
Jelfa, Jelenia Góra, Poland; Nimbex, GlaxoSmithKline, Brentford, UK) performed at the follicular phase of the menstrual cycle (between days 9 and 12 of the cycle). The study population consisted of 22 females with histopathologically proven endometriosis and the control group consisted of 20 females with unexplained infertility and no evidence of pelvic pathology. The severity of endometriosis was scored according to the revised American Society for Reproductive Medicine (rASRM). The present study included 15 patients with minimal endometriosis (I/II rASRM) and 7 patients with advanced stage endometriosis (III/IV rASRM). All patients had regular menstrual cycles. Clinical data regarding the patients are shown in Table I.

Isolation of mononuclear cells. $\mathrm{PB}$ and $\mathrm{PF}$ were diluted using phosphate-buffered saline (PBS) without $\mathrm{Ca}^{2+}$ and $\mathrm{Mg}^{2+}$ ions at a 1:1 ratio. The prepared solutions were layered on Gradisol L density gradient medium (Aqua-Med, Łódź, Poland) and centrifuged for $20 \mathrm{~min}$ at $700 \mathrm{x}$ g. Following centrifugation, the obtained interphase was transferred into a test tube using a Pasteur pipette and was washed twice using PBS without $\mathrm{Ca}^{2+}$ and $\mathrm{Mg}^{2+}$ ions. The supernatant was discarded and the precipitate containing mononuclear cells was resuspended in $1 \mathrm{ml}$ RPMI-1640 following washing.

Treg detection. Tregs in the PB and PF were assessed by analyzing the expression of the CD4 and CD25 cell surface antigens, as well as the expression of the intracellular FOXP3 antigen using a BD FACSCalibur ${ }^{\mathrm{TM}}$ flow cytometer (BD Biosciences, San Jose, CA, USA). The percentage of CD4 ${ }^{+}$ $\mathrm{CD}_{25}{ }^{+} \mathrm{FOXP}^{+}$Tregs in the $\mathrm{CD}^{+} \mathrm{T}$ lymphocyte subpopulation was determined using the Human Treg Flow $^{\mathrm{TM}}$ kit (FOXP3 Alexa Fluor ${ }^{\circledR}$ 488/CD4 PE-Cy5/CD25 PE) purchased from BioLegend (San Diego, CA, USA).

Measurement of CA-125 levels in serum samples. Serum samples were taken from the antecubital vein, prior to general anesthesia. The CA125 concentration was measured using an ADVIA Centaur CA125 (Siemens, Deerfield, IL, USA) immunodiagnostic assay using a chemiluminometric technique. The assay was performed using the ADVIA Centaur XP system (Siemens, Malvern, PA, USA).

Determination of white blood cell count and lymphocyte count. Peripheral blood was taken from the antecubital vein, prior to general anesthesia. A peroxidase method was used to determine the white blood cell and lymphocyte counts in the PB using an ADVIA 2120 system (Siemens).

Statistical analysis. Statistical analysis was performed using Statistica 7.1 software (Statsoft Inc., Tulsa, OK, USA. The normal distribution of each variable within the groups was assessed using the Shapiro-Wilk test. Normal distribution was not observed, thus Mann-Whitney-U tests were used for the statistical comparison between PB and PF in the groups of patients. The Wilcoxon-paired test was used to compare the PB and PF. The Spearman rank correlation coefficient was used for correlation analyses. Data are presented as the mean \pm standard deviation. $\mathrm{P}<0.05$ was considered to indicate a statistically significant difference.

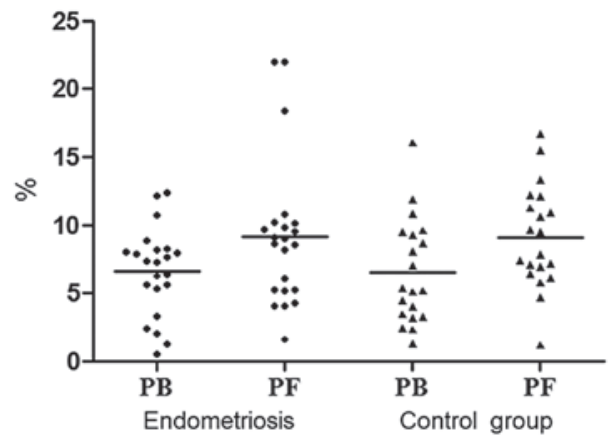

Figure 1. Percentage of Tregs among $\mathrm{CD}^{+}$lymphocytes in the $\mathrm{PB}$ and $\mathrm{PF}$ in patients with endometriosis and the control group. PB, peripheral blood; $\mathrm{PF}$, peritoneal fluid; Tregs, $\mathrm{T}$ regulatory cells; $\mathrm{CD}$, cluster of differentiation.

Ethics statement. The present study was approved by the Ethics Committee of the Medical University of Lublin (no. KE-0254/244/2011; Lublin, Poland). Written informed consent was obtained from all patients.

\section{Results}

Clinical data of the patients in the endometriosis and control groups. Clinical characteristics of the patients in the endometriosis and control groups are presented in Table I. The average age of the patients in the endometriosis and control groups was $33.58 \pm 4.74$ and $31.24 \pm 5.95$ years, respectively. No significant difference was observed in the body mass index in the patients with endometriosis $\left(22.33 \pm 3.45 \mathrm{~kg} / \mathrm{m}^{2}\right)$ compared with those in the control group $\left(23 \pm 1.79 \mathrm{~kg} / \mathrm{m}^{2}\right)$. Furthermore, blood cell and lymphocyte counts in the PB were not found to be significantly different between the two groups. However, the CA125 serum concentration was observed to be significantly higher in the patients in the endometriosis group $(58.98 \pm 80.33 \mathrm{mIU} / \mathrm{ml})$ compared with that in the patients in the control group $(34.12 \pm 80.56 \mathrm{mIU} / \mathrm{ml} ; \mathrm{P}<0.05)$.

Tregs in the PF and PB in the endometriosis and control groups. The quantity of Tregs among the $\mathrm{CD} 4^{+}$lymphocytes in the PF compared with the PB in the endometriosis and control groups is shown in Figs. 1 and 2. The percentage of Tregs in the PF (endometriosis group, 9.1\% \pm 5.4 ; and control group, $9.1 \% \pm 3.8$ ) was observed to be higher than in the PB (endometriosis group, $6.5 \% \pm 3.2$; and control group, $6.5 \% \pm 3.7)$ in the two groups. No significant difference was observed in the percentage of Tregs in the PF $(9.1 \% \pm 5.4)$ compared with that in the PB $(6.5 \pm 3.2 \%)$ in the patients in the endometriosis group $(\mathrm{P}>0.05)$. However, the percentage of Tregs in the PF $(9.1 \% \pm 3.8)$ was found to be significantly higher than that in the PB $(6.5 \% \pm 3.7)$ in the patients in the control group $(\mathrm{P}=0.002)$.

Analysis of Tregs in various stages of endometriosis. The percentage of Tregs in the PB and PF of patients with various stages of endometriosis is shown in Fig. 2. The percentage of Tregs in the patients with I/II rASRM endometriosis was found to be $8.8 \pm 4.4 \%$ in the PF and $6.9 \pm 3.2 \%$ in the PB, which was not a statistically significant difference $(\mathrm{P}>0.05)$. Furthermore, no significant difference was observed in the 
Table I. Clinical data regarding the patients included in the endometriosis and control groups.

\begin{tabular}{|c|c|c|c|}
\hline Parameter & Endometriosis group & Control group & P-value \\
\hline Age (years) & $33.58 \pm 4.74$ & $31.2 \pm 5.9$ & NS \\
\hline $\mathrm{BMI}\left(\mathrm{kg} / \mathrm{m}^{2}\right)$ & $22.33 \pm 3.45$ & $23.1 \pm 1.8$ & NS \\
\hline Serum CA125 concentration (mIU/ml) & $58.98 \pm 80.33$ & $34.1 \pm 80.6$ & $<0.05$ \\
\hline White blood cells ( $\mathrm{x} 10^{3}$ cells $\left./ \mathrm{ml}\right)$ & $7.53 \pm 2.31$ & $6.8 \pm 1.8$ & NS \\
\hline Lymphocytes $\left(\mathrm{x} 10^{3}\right.$ cells/ml) & $1.63 \pm 0.44$ & $1.7 \pm 0.3$ & NS \\
\hline
\end{tabular}

Data are presented as the mean \pm standard deviation. BMI, body mass index; NS, not significant.

Table II. Analysis of the percentage of T regulatory cells in the PF and PB in patients with mild endometriosis compared with the control group.

\begin{tabular}{lccc}
\hline Parameter & Endometriosis I/II rASRM (\%) & Control group (\%) & P-value \\
\hline PF & $8.8 \pm 4.4$ & $9.1 \pm 3.8$ & 0.68 \\
PB & $6.9 \pm 3.2$ & $6.5 \pm 3.7$ & 0.60 \\
\hline
\end{tabular}

Data are presented as the mean \pm standard deviation. PF, peritoneal fluid; PB, peripheral blood; rASRM, revised American Society for Reproductive Medicine.

Table III. Analysis of the percentage of T regulatory cells in the PF and PB in patients with advanced endometriosis compared with the control group.

\begin{tabular}{lccr}
\hline Parameter & Endometriosis III/IV rASRM (\%) & Control group (\%) & P-value \\
\hline PF & $9.9 \pm 7.4$ & $9.1 \pm 3.8$ & 0.72 \\
PB & $5.7 \pm 3.1$ & $6.5 \pm 3.7$ & 0.53 \\
\hline
\end{tabular}

Data are presented as the mean \pm standard deviation. PF, peritoneal fluid; PB, peripheral blood; rASRM, revised American Society for Reproductive Medicine.

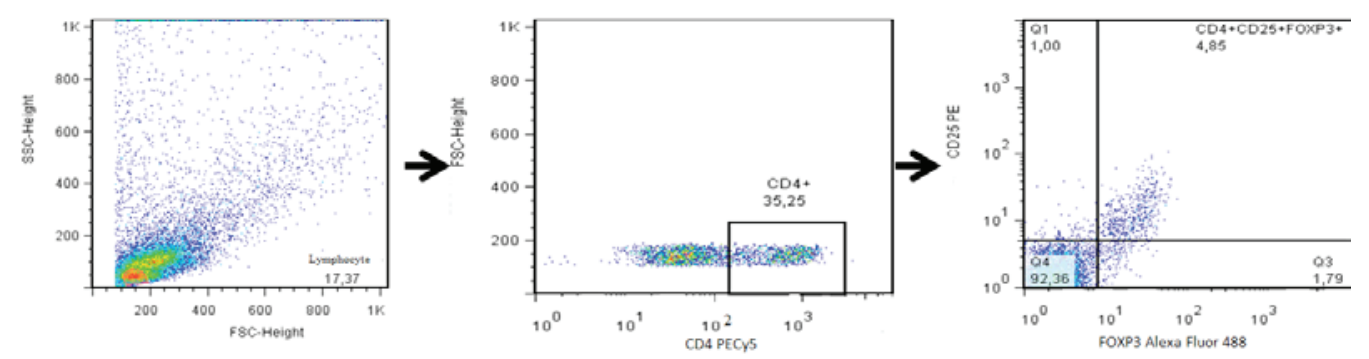

Figure 2. Flow cytometric analysis of T regulatory cells. Lymphocytes were gated on the basis of forward scatter vs. side scatter signals. CD4+ lymphocytes were then gated and the proportion of $\mathrm{CD} 25^{+} \mathrm{FOXP}^{+}$cells among the $\mathrm{CD} 4^{+}$cells was estimated. $\mathrm{CD}$, cluster of differentiation.

percentage of Tregs in the PF $(9.9 \pm 7.4 \%)$ compared with that in the PB $(5.7 \pm 3.1 \%)$ in the patients with III/IV rASRM $(\mathrm{P}>0.05)$. The percentage of Tregs in the patients with I/II rASRM endometriosis compared with those with III/IV rASRM endometriosis was not found to be significantly different in the PB or the PF. Table II shows the analysis of the Tregs in the PF and PB of the patients with mild
(I/II rASRM) endometriosis compared with those in the control group. No significant difference was observed in the percentage of Tregs in the $\mathrm{PF}(\mathrm{P}=0.6)$ or the $\mathrm{PB}(\mathrm{P}=0.68)$ in the patients with mild endometriosis (I/II rASRM) compared with those in the control group (Table II). Moreover, no significant difference was found in the PF $(\mathrm{P}=0.72)$ or the $\mathrm{PB}(\mathrm{P}=0.53)$ in the patients with advanced endometriosis 


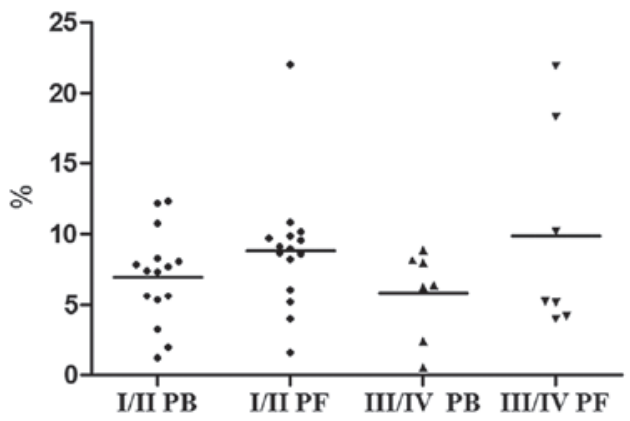

Figure 3. Percentage of Tregs among $\mathrm{CD} 4^{+}$lymphocytes in the PB and PF in patients with mild and advanced stage endometriosis. $\mathrm{PB}$, peripheral blood $\mathrm{PF}$, peritoneal fluid; Tregs, $\mathrm{T}$ regulatory cells; $\mathrm{CD}$, cluster of differentiation.

(III/IV rASRM) compared with those in the control group (Table III).

Correlation between Tregs and CA125, white blood cell count and lymphocyte count. No significant correlation was observed between the percentage of Tregs in the PF or the PB and the CA125 levels, white blood cell count and lymphocyte count (data not shown).

\section{Discussion}

The first study to report immune changes during endometriosis was published in 1989 by Startseva and Shvetsov (13). At present, there is considerable evidence to support the role of the immune system in the development of endometriosis. The immune theory proposed by Braun and Dmowski (2) is widely accepted and states that endometriosis is caused by hereditary or acquired immune deficiency. This theory is important for understanding the pathogenesis of the disease.

Oosterlynck et al (14) identified quantitative and qualitative changes in the immune cells within the PF of females with endometriosis. Furthermore, Steele et al (15) found that deficient cellular immunity may result in an inability to recognize the presence of endometrial tissue in abnormal locations. Dmowski et al (16) identified an increased concentration of leukocytes and macrophages in the peritoneal cavity and the ectopic endometrium in patients with endometriosis and reported that endometrial cell proliferation is stimulated by peripheral blood monocytes and inhibited by peritoneal macrophages. The cytotoxic effect of peritoneal macrophages was found to be inversely correlated with the stage of endometriosis. Moreover, endometrial cells in patients with endometriosis were observed to be more sensitive to the stimulatory effect of peripheral blood monocytes and were found to be more resistant to the cytotoxicity of immune cells (16). Oosterlynck et al (14) also reported that NK cell activity may be reduced in patients with endometriosis, resulting in decreased cytotoxicity to the autologous endometrium.

The present study assessed the presence of Tregs in patients with endometriosis. In a previous study, Jasper et al (17) identified that FOXP3 mRNA expression was decreased in the endometrial tissue of infertile patients during the secretory phase. Furthermore, Berbic et al (18) found that the concentration of Tregs was reduced in the endometrium of females with endometriosis during the secretary phase. Endometriosis is frequently associated with infertility; therefore, identifying the correlation between infertility and the percentage of FOXP3 ${ }^{+}$ cells may be promising for future infertility therapy (18). It has been reported that patients with endometriosis exhibit an increased number of Tregs in the endometrium during the secretory phase, which may lead to Treg-mediated immunosuppression and decreased dendritic cell maturation through the downregulation of the ability of DCs to effectively present antigens, which may facilitate the survival of shed endometrial fragments. Berbic et al (18) demonstrated that a number of immune cell populations, including macrophages, NK cells and $\mathrm{T}$ cells are recruited to the core zone of endometriotic lesions.

Berbic et al (18) also revealed no significant difference in the percentage of Tregs in the eutopic endometrium during the proliferative phase in females with endometriosis compared with those without endometriosis. The findings of the present study are in accordance with this, as no significant difference was found in the percentage of Tregs in the PF or the PB in the patients in the endometriosis group compared with those in the control group. Moreover, Berbic et al (18) found no significant difference in the expression of Foxp3 in the ectopic endometrium compared with that in the eutopic endometrium at menstrual, proliferative or secretory phases of the cycle (18). A higher number of Tregs were observed in the ectopic endometrium compared with the eutopic endometrium, but FoxP3 ${ }^{+}$ Tregs were only found in $31 \%$ of the peritoneal lesions. This suggests that FOXP3 expression is likely to correlate with the stage of lesion development and progression (18). The severity of the symptoms in endometriosis does not necessarily correlate with the progression of the disease. It has been reported that advanced endometriosis may be asymptomatic, while early stages of the disease may correlate with severe symptoms. However, to the best of our knowledge, Tregs in the PB and PF in patients with varying endometriosis severity have not been investigated. The present study identified no significant difference in the percentage of Tregs in the patients with mild endometriosis compared with those with advanced endometriosis, although a higher percentage of Tregs was found in the PF in the two groups. Thus, in the present study, changes in the percentage of peritoneal Tregs did not depend on the stage of endometriosis.

It has also been reported that the percentage of FoxP3 positive lymphocytes within the subpopulation of T cells in the $\mathrm{PB}$ of females with ovarian endometriosis does not differ from that observed in healthy females (19). In healthy, fertile females, the significant decrease in Tregs in the PB typically occurs immediately subsequent to ovulation. Thus, the increase in the number of Tregs may depend on estrogen levels and estrogen may also be responsible for the increase in the immune suppressive potential of these cells. Furthermore, local upregulation of estrogen production has been found to induce Treg proliferation in females with ovarian endometrioma (20).

The suppression of the local immune response using a Treg-dependent mechanism may prevent clearing of ectopic tissues within the peritoneal microenvironment (21). The present study identified a higher concentration of Tregs in the PF compared with the PB in females with endometriosis and those in the control group. Thus, the local peritoneal-specific immune response may be different to the systemic immune 
response. Ectopic endometrial tissues have been found to express cytochrome p450; therefore, they have the capacity to produce estrogen, which may influence PF Treg concentration. In the present study, the excessive local immune response may interfere with fertility in the patients in the two groups. The increase in Tregs found in the PF compared with the PB may reflect a local host-defense mechanism in the patients and may be involved in the pathogenesis of endometriosis. Thus, the body's immune response to endometriosis may be local rather than general.

In a meta-analysis performed by Mol et al (22), the routine use of CA125 determination in subfertile patients was found to identify a subgroup likely to benefit from laparoscopy. It has also been reported that a screening method combining CA125 with the level of a leukocyte subpopulation increases the positive predictive values in detecting endometriotic patients (23). In the present study, no significant correlation was found between CA125 serum level and Treg percentage in the patients in the endometriosis or control groups. Furthermore, to the best of our knowledge, no correlation has previously been reported between Tregs in the PF or PB and the concentration of CA125, white blood cell count or lymphocyte count. These findings suggest that endometriosis is associated with local manifestations; however, the specific role of these modulations has yet to be elucidated.

Nothnick (6) reported that endometriosis is similar to autoimmune disorders, with Tregs being associated with autoimmunity. In the present study, the percentage of Tregs in the $\mathrm{PF}$ was found to be significantly higher than that in the PB in the patients in the control group, but not in the patients in the endometriosis group. Thus, the local host-defense mechanisms may exist in healthy females, but may be impaired in those with endometriosis. Therefore, endometriosis should not be treated as an autoimmune condition and Tregs may be a potential target for the treatment of endometriosis.

In conclusion, the increase in Tregs in the PF in healthy females reflects a host-defense mechanism. This may suggest that local host-defense mechanisms and immune responses are deficient in patients with endometriosis. Therefore, endometriosis should not be treated as an autoimmune condition.

\section{Acknowledgements}

The present study was supported by a grant from Lublin Medical University (grant no. DS 326/2013).

\section{References}

1. Olive DL and Schwartz LB: Endometriosis. N Engl J Med 328: 1759-1769, 1993

2. Braun DP and Dmowski WP: Endometriosis: abnormal endometrium and dysfunctional immune response. Curr Opin Obstet Gynecol 10: 365-369, 1998.
3. Vinatier D, Dufour P and Oosterlynck D: Immunological aspects of endometriosis. Hum Reprod Update 2: 371-384, 1996.

4. Matarese G, De Placido G, Nikas Y and Alviggi C: Pathogenesis of endometriosis: natural immunity dysfunction or autoimmune disease?. Trends Mol Med 9: 223-228, 2003.

5. Giudice LC and Kao LC: Endometriosis. Lancet 364: 1789-1799, 2004.

6. Nothnick WB: Treating endometriosis as an autoimmune disease. Fertil Steril 76: 223-231, 2001.

7. Sakaguchi S, Sakaguchi N, Asano M, Itoh M and Toda M: Immunologic self-tolerance maintained by activated $\mathrm{T}$ cells expressing IL-2 receptor alpha-chains (CD25). Breakdown of a single mechanism of self-tolerance causes various autoimmune diseases. J Immunol 155: 1151-1164, 1995.

8. Sakaguchi S, Yamaguchi T, Nomura T and Ono M: Regulatory T cells and immune tolerance. Cell 133: 775-787, 2008.

9. Nandakumar S, Miller CW and Kumaraguru U: T regulatory cells: an overview and intervention techniques to modulate allergy outcome. Clin Mol Allergy 7: 5, 2009.

10. Sakaguchi S, Ono M, Setoguchi R, et al: Foxp3 $3^{+} \mathrm{CD} 25^{+} \mathrm{CD} 4^{+}$ natural regulatory $\mathrm{T}$ cells in dominant self-tolerance and autoimmune disease. Immunol Rev 212: 8-27, 2006.

11. Rouse BT: Regulatory T cells in health and disease. J Intern Med 262: 78-95, 2007

12. Kondělková K, Vokurková D, Krejsek J, Borská L, Fiala Z and Ctirad A: Regulatory T cells (TREG) and their roles in immune system with respect to immunopathological disorders. Acta Medica (Hradec Kralove) 53: 73-77, 2010.

13. Startseva NV and Shvetsov MV: The natural cellular cytotoxicity of endometriosis patients. Akush Ginekol (Mosk) 11: 58-60, 1989 (In Russian)

14. Oosterlynck DJ, Cornillie FJ, Waer M, et al: Women with endometriosis show a defect in natural killer activity resulting in a decreased cytotoxicity to autologous endometrium. Fertil Steril 56: 45-51, 1991.

15. Steele RW, Dmowski WP and Marmer DJ: Immunologic aspects of human endometriosis. Am J Reprod Immunol 6: 33-36, 1984.

16. Dmowski WP, Gebel HM and Braun DP: The role of cell-mediated immunity in pathogenesis of endometriosis. Acta Obstet Gynecol Scand Suppl 159: 7-14, 1994.

17. Jasper MJ, Tremellen KP and Robertson SA: Primary unexplained infertility is associated with reduced expression of the t-regulatory cell transcription factor Foxp3 in endometrial tissue. Mol Hum Reprod 12: 301-308, 2006.

18. Berbic M, Hey-Cunningham AJ, Ng C, et al: The role of Foxp3 $3^{+}$ regulatory T-cells in endometriosis: a potential controlling mechanism for a complex, chronic immunological condition. Hum Reprod 25: 900-907, 2010.

19. Budiu RA, Diaconu I, Chrissluis R, Dricu A, Edwards RP and Vlad AM: A conditional mouse model for human MUC1-positive endometriosis shows the presence of anti-MUC1 antibodies and Foxp3 $^{+}$regulatory T cells. Dis Model Mech 2: 593-603, 2009.

20. Zeitoun K, Takayama K, Sasano H, et al: Deficient 17-hydroxysteroid dehydrogenase type 2 expression in endometriosis: failure to metabolize 17beta-estradiol. J Clin Endocrinol Metab 83: 4474-4480, 1998.

21. Prieto GA: Progression of endometriosis to cancer: too MUCh FoxP $3^{+}$regulatory T-cell response? Dis Model Mech 4: 139-140, 2011.

22. Mol BW, Bayram N, Lijmer JG, Wiegerinck MA, Bongers MY, van der Veen F and Bossuyt PM: The performance of CA-125 measurement in the detection of endometriosis: a meta-analysis. Fertil Steril 70: 1101-1108, 1998

23. Gagné D, Rivard M, Pagé M, et al: Development of a nonsurgical diagnostic tool for endometriosis based on the detection of endometrial leukocyte subsets and serum CA-125 levels. Fertil Steril 80: 876-885, 2003. 